\title{
Modulation of early ERP components with peripheral precues: a trend analysis
}

\author{
Rob H.J. van der Lubbe*, Jaap C. Woestenburg \\ Cognitive Psychology, Free University, De Boelelaan 1111, $1081 \mathrm{HV}$, Amsterdam, The Netherlands
}

\begin{abstract}
In the visual domain, involuntary allocation of attentional resources can be induced by using peripheral cues. An enlargement of the P1 ERP-component has been reported in connection with voluntary allocation of resources induced by symbolic cues, but until recently, it has not been reported in connection with involuntary allocation of resources. However, involuntary allocation of resources was only investigated with long cue-target intervals (SOAs) of about $600 \mathrm{~ms}$. Therefore, an experiment was conducted with SOAs between 100 and $300 \mathrm{~ms}$. After a $100 \%$ valid peripheral cue a bilateral multi-item array was presented. Trend analyses, which were employed to estimate the ERP elicited by the array corrected for linear and nonlinear contribution of the cue, showed a contralateral enhancement for the posterior P150 and the N230 component. Hence, involuntary allocation of resources with short SOAs might invoke the same level as voluntary allocation of resources. The P150 enhancement may be interpreted as a reflection of allocated resources at a specific location, whereas the N230 enhancement might reflect elaborated processing at the contralateral hemisphere. 1997 Elsevier Science B.V.
\end{abstract}

Keywords: Visual selective attention; Involuntary allocation of attentional resources; ERPs; The P1 component; Peripheral precues; Trend analysis

\footnotetext{
* Corresponding author. Tel.: + 3120 4448799; fax: + 3120 4448832; e-mail: robvdl@psy.vu.nl.
} 


\section{Introduction}

When subjects are instructed to focus their attention on a cued location in visual space, their reactions with respect to targets presented at the cued location are faster than to targets presented at uncued locations. In terms of mental resource models, either a specific or a nonspecific source of energy is allocated to a particular cognitive operation. Thus, within this perspective, it may be suggested that attention is a mechanism that allocates extra capacity or resources at a certain level of information processing (e.g., at the level of feature extraction, memory rehearsal, or response selection). Two main views have been put forward with respect to the processing level(s) at which resources can be allocated. The first view presumes that resources are allocated at an early sensory processing level (Jonides, 1983; Posner, 1980). For instance, Jonides (1983) argued that focussing attention at a specific location results in an allocation of resources on a particular spatial locus, which may compensate for the limitations of the visual system. The second view presumes that resources are allocated at later stages of information processing (e.g., task-specific decision making, memory rehearsal, or response selection (Duncan, 1980; Shaw, 1984)). Recently, event-related potentials (ERP) studies provided strong evidence for the view that resources can be allocated at an early sensory level (Hawkins et al., 1990; Mangun \& Hillyard, 1990, 1991). Resources can be allocated either voluntarily (controlled) or involuntarily (automatically). If we assume that resources can be allocated at an early sensory processing level, then the following question can be raised: do voluntary and involuntary allocation of attentional resources invoke a similar level of sensory processing as reflected in ERP modulations?.

Voluntary allocation of resources is assumed to be manifest in conditions with symbolic cues (Jonides, 1981). On the basis of the cue, subjects focus their attention in a controlled way on a specific location. Usually, a centrally located arrow is used as cue that has a specific 'cue validity'. 'Cue validity' means that the cue indicates the correct target location on a specific proportion of the trials, and it indicates an incorrect location on the remaining proportion of trials. For instance, a cue validity of $75 \%$ means that for $75 \%$ of the trials, the cue provides valid information concerning the location of the target, and for $25 \%$ of the trials, the cue provides invalid information. An attention effect can be estimated by making a comparison between the results of validly and invalidly cued trials (i.e., cost-benefit analysis).

Jonides (1981) provided evidence for the view that peripheral cues, such as dots or stripes, automatically attract attention. Thus, involuntary allocation of resources can be demonstrated in conditions with peripheral cues that appear either near a relevant (validly cued) or near an irrelevant (invalidly cued) position. Posner \& Cohen (1984) suggested that peripheral cues not only automatically attract attention, but also induce an inhibitory effect - i.e. inhibition of return (IOR) - with long cue-target intervals: stimulus onset asynchronies (SOAs). Hence, involuntary allocation of resources to peripheral onsets may be a short-lived phenomenon.

Müller \& Rabbitt (1989), proposed a two-mechanism model of spatial orienting with a fast-acting mechanism that is involuntarily triggered by peripheral onsets 
(independent of cue validity) and a slow-acting mechanism that is voluntarily triggered (dependent on cue validity). At long stimulus onset asynchronies (SOAs: cue-target onset intervals; $>400 \mathrm{~ms}$ ) both peripheral and symbolic cues are assumed to be involved in the same slow-acting mechanism. Hence, for long SOAs both symbolic and peripheral cues depend on cue validity. Interestingly, no differences were found between peripheral and symbolic cues for long SOAs. It may be suggested that the inhibition effect as reported by Posner \& Cohen (1984) can be suppressed by the slow-acting mechanism. Thus, symbolic cues can trigger a slow-acting mechanism whereas peripheral cues can trigger both a slow- and a fast-acting mechanism, and the slow-acting mechanism may overrule inhibition of return induced by peripheral onsets when relatively long SOAs are used.

\section{Event related potential (ERP) studies}

Mangun \& Hillyard (1991) and Heinze et al. (1990) focused on the effect of voluntary allocation of resources on ERPs. In the study of Mangun \& Hillyard (1991), arrows indicated the side of a subsequently presented target with a cue validity of $80 \%$ and an SOA of $800 \mathrm{~ms}$. In a first task, where identification of the target was task relevant, an enhanced occipital P1 (90-130 ms) and N1 (150-200) component were found for validly as compared to invalidly cued targets. The P1 enhancement was first evident over the contralateral hemisphere, and later over the ipsilateral hemisphere, whereas the N1 enhancement was larger over the ipsilateral hemisphere. In a second task, in which the target had only to be detected, an enhanced nonlateralized Pl component but no enhanced $\mathrm{N} 1$ component was observed for validly as compared to invalidly cued targets. Heinze et al. (1990) also reported differences between the $\mathrm{Pl}$ and $\mathrm{N} 1$ modulation with bilateral stimulus arrays. They suggested that enhancement of the $\mathrm{P} 1$ component reflects facilitation of the visual pathways, whereas enhancement of the N1 component reflects 'further orienting' to target positions. In terms of the spotlight metaphor (Posner et al., 1980), modulation of the Nl component might reflect the process that guides the spotlight, whereas modulation of the P1 component might reflect an increase in sensitivity at the attended region. Speaking more generally, modulation of the N1 component might reflect a process that allocates, or reallocates attentional resources to a specific spatial location, whereas modulation of the Pl component might reflect the consequence of allocated resources on sensory processing.

ERP studies addressing the fast-acting involuntarily triggered mechanism elicited by peripheral cues, have been reported by Hillyard et al. (1994) and Eimer (1994). Hillyard et al. performed an ERP study in which they compared the effects of symbolic (a centrally located arrow) and peripheral cues (four peripherally presented dots) in an identification task, with identical SOAs $(600-800 \mathrm{~ms})$ and a cue validity of $75 \%$. Both types of cues showed an enhancement of the N1 (150-200 $\mathrm{ms}$ ) and N2 (250-300 ms) component for validly as compared to invalidly cued targets at occipital, temporal, parietal and central sites. For symbolic cues, a P1 enhancement was observed for validly as compared to invalidly cued targets, 
however, no Pl effect was found for peripheral cues. Hillyard et al. suggested that peripheral and symbolic cues differ in their capacity to invoke the earliest form of attentional selection.

On the basis of the result of this study it may be suggested that both voluntary and involuntary allocation of attentional resources modulate processing at an early sensory level which is reflected in a posterior modulation of the P1 component for symbolic cues and the N1 component for peripheral cues. However, is it plausible to believe that attention operates at a later moment in time with peripheral cues, as was suggested by Hillyard et al.? Inhibition of return (Posner \& Cohen, 1984) may be responsible for not finding a modulation of the $\mathrm{P} 1$ component for peripheral cues because relatively long SOAs were used. However, behavioral measures in the task of Hillyard et al. showed no indication of IOR. It must be noted that according to Müller \& Rabbitt (1989) long SOAs for peripheral cues involves the slow-acting mechanism instead of the fast-acting mechanism. Therefore, the use of short SOAs might reveal an effect on the early Pl component when attention is involuntarily oriented by peripheral cues. However, using short SOAs will yield a methodological problem, as it becomes difficult to separate ERPs elicited by the cue and the target.

To test this hypothesis, we decided to use a design with various intervals between the peripheral cue and the target stimulus. The orthogonal polynomial trend analysis (OPTA; Woestenburg et al., 1983) was employed to separate the contribution of the ERP elicited by the cue from the ERP elicited by the imperative stimulus (see Section 3, and for an alternative method see Woldorff (1993)).

The manipulation of cue validity to perform cost-benefit analysis may complicate the interpretation of ERPs. Van der Heijden (1992) argued that cost-benefit analysis might favor a probability matching strategy in which subjects only attend to a precued location on a specific proportion of the trials. In addition, subjects might prepare less than optimally with $75 \%$ valid cues as compared to $100 \%$ valid cues. Therefore, we decided to use $100 \%$ valid cues. We also used bilateral multiple-item arrays with laterally presented targets to enable inspection of hemispherical differences (contralateral versus ipsilateral activity). Higher activity in the contralateral hemisphere suggests that the side of the target at that moment in time is processed more extensively. The comparison of ipsilateral and contralateral activity provides a way to control for the possibility of a physiologically refractory effect (i.e. a decrease in sensitivity for the involved neuronal population) resulting from the presentation of a peripheral cue. If such effects play an important role then one would expect an amplitude reduction at the contralateral hemisphere due to the cue being mainly processed by this hemisphere. However, this effect will be temporarily related to the onset of the cue and not the array. After using the OPTA procedure, the refractory effect will not be included in the estimation of the ERP elicited by the array. In conclusion, an important difference between the study of Hillyard et al. (1994) and this study is that we will focus on differences in hemispheric processing instead of differences between validly and invalidly cued trials, and we will use much shorter SOAs. 


\section{Experimental methods}

\subsection{Subjects}

Eight right-handed subjects (all normal or corrected to normal vision, four male, mean age 22 years) participated. They received, depending on the amount of hours (about 6) they co-operated in this part of a larger experiment, about 90 Dutch guilders $(\sim$ US $\$ 60)$.

\subsection{Stimuli and task}

The stimuli (Fig. 1) consisted of black arrays of six elements (each $0.69^{\circ} \times 0.69^{\circ}$ ) presented for $750 \mathrm{~ms}$ on a white screen. Five elements were identical distractors and one was a deviant target. The distractors ( + 's) and targets (distractors missing either an upper or lower part) covered an equal part of the left and right visual hemifield. The elements were located at an eccentricity (distance, in degrees, to the fixated position) of $3.1^{\circ}, 1.9^{\circ}$ and $0.7^{\circ}$ to the left and right side of a fixation square $\left(0.53^{\circ} \times 0.38^{\circ}\right)$ that was continuously present. The targets were only presented at either $0.7^{\circ}$ or $3.1^{\circ}$ to the left or right side of the fixation square, yielding four possible target positions. The arrays were preceded by a black underscore $\left(0.69^{\circ} \times\right.$ $0.23^{\circ}$ ), regarded as cue, that validly indicated the precise target position. They were presented $0.63^{\circ}$ below the arrays for a duration of $100 \mathrm{~ms}$. SOA varied between 100 and $300 \mathrm{~ms}$ in equal steps of $10 \mathrm{~ms}$ resulting in 21 SOA blocks. The inter-trial interval amounted to $2560 \mathrm{~ms}$.

Subjects had to fix on the fixation square during a block of 80 trials. It was emphasized that they should minimize eye movements. Furthermore, they were informed about the directional aspect of the cue. Subjects were instructed to press

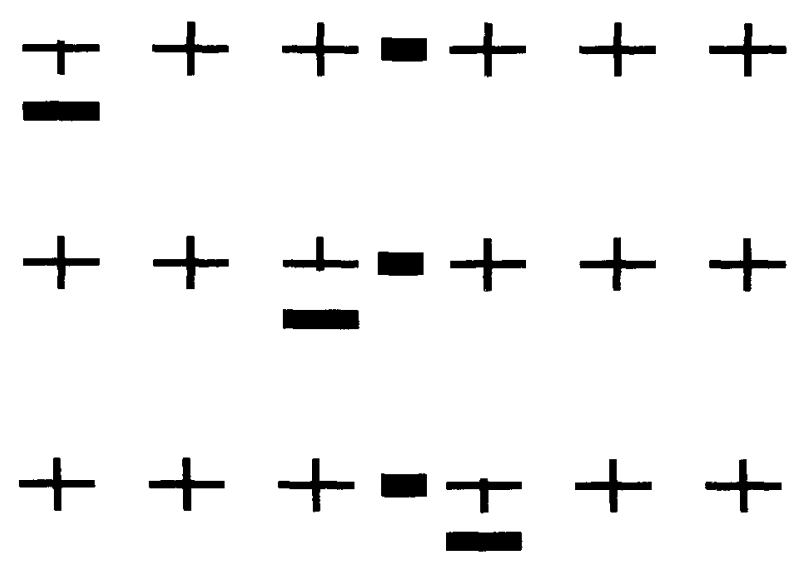

Fig. 1. Arrays consisting of six elements, five distractors (plus signs), and one of two possible targets (missing the upper or lower stroke of the plus sign). Targets could appear at one out of four possible positions, and were preceded by a peripheral cue (black bar) indicating the precise position of the target. 
a left or right key with their left or right hand upon arrival of the target. In one session, subjects were instructed to press a left key for the target missing the lower stroke and to press a right key for the target missing the upper stroke. In another session this target response relation was reversed. Equal emphasis was given to speed and accuracy.

\subsection{Design and procedure}

A within-subjects repeated measures design was used with ERP components, vertical and horizontal electro-oculogram (VEOG and HEOG), reaction times (RTs), and proportion errors (PEs) as dependent variables. For each subject, the experiment was conducted on three separate, but not consecutive days. Each $\mathbf{S}-\mathbf{R}$ mapping session consisted of 21 SOA blocks (SOA was varied between blocks), each containing 80 trials in which targets randomly appeared at one of four possible positions. The order of SOA blocks was varied pseudorandomly. Three practice blocks were carried out at the beginning of each day, and again after a switching of the target response relation. The order of $\mathrm{S}-\mathrm{R}$ mapping was counterbalanced between subjects. Eye movements were calibrated to exclude trials in which EOG exceeded a prespecified criterion.

\subsection{Apparatus and recordings}

Stimulus presentation and data collection (electro-encephalo-gram (EEG), VEOG and HEOG, RTs and PEs) were controlled by the InstEP system (Campbell \& Bell, 1992) running on a 386 SX (slave) and a 486 DX2 (master) computer. Subjects were lying on a bed in a sound-attenuated dimly lit (15 lux) cubicle. On each side of the bed a response button was positioned at an optimal distance for each subject. Stimuli were presented on a screen placed at a distance of $75 \mathrm{~cm}$ from the subject. HEOG and VEOG were measured by two tin electrodes placed at the outer canthus of each eye and by two tin electrodes placed infra-orbital and supra-orbital in line with the pupil. EEG was recorded from nonpolarizable tin electrodes mounted in an elastic cap (Electro Cap International) and located at standard left and right hemisphere positions spanning the surface of the scalp (International 10/20 System names: F3, F4, C3, C4, P3, P4, T5, T6, O1, O2. Two extra electrodes were mounted on the cap, OL, between $\mathrm{T} 5$ and $\mathrm{O} 1$, and $\mathrm{OR}$, between T6 and O2 (Mangun \& Hillyard, 1990). Linked ear electrodes were used as reference electrodes. Recording started $150 \mathrm{~ms}$ before the onset of the cue and continued for $2560 \mathrm{~ms}$. The signals were amplified with a lowpass filter of $30 \mathrm{~Hz}$ and a time constant of $5 \mathrm{~s}$, and were digitized at $100 \mathrm{~Hz}$.

\subsection{EEG analysis}

First, InstEP files were converted to the STPBS format (Woestenburg, 1994) for further data analysis and data processing. EEGs of all SOA blocks were shifted until the imperative stimulus appeared at time point ' 0 ' which created a prestimulus 
interval of minimally $250 \mathrm{~ms}$. Trials containing horizontal $(>40 \mu \mathrm{V})$ and vertical eye movements $(>500 \mu \mathrm{V}), 0-400 \mathrm{~ms}$ after onset of the array, were removed. Preaveraging was performed on correct responses for eight categories, two possible targets appearing at four possible positions, for each SOA block. Per eccentricity, the preaverages were sorted in separate files for targets appearing at the left and right. In the left target files polarity of the HEOG was reversed (for an additional checking procedure), and the order of the EEG channels was adjusted (i.e.. F3 vs. $\mathrm{F} 4, \mathrm{C} 3$ vs. $\mathrm{C} 4, \mathrm{~T} 5$ vs. T6, OL vs. OR, and $\mathrm{O} 1$ vs. $\mathrm{O} 2$ were exchanged). A new average per SOA block was computed for the reversed left and the non-reversed right file, resulting in two eccentricity categories $\left(0.7^{\circ}\right.$ and $\left.3.1^{\circ}\right)$. This procedure created an average over both hemispheres for contralateral and ipsilateral activity. These averages were ordered from a short $(100 \mathrm{~ms})$ to a long $(300 \mathrm{~ms})$ SOA. Subsequently, the OPTA (Woestenburg et al., 1983; Woestenburg, 1994) was performed for each subject.

In many ERP studies, it is assumed that a single trial EEG consists of a fixed component and a random noise component. However, in our study the ERP elicited by the target not only consists of a fixed component and a random noise component, but also of a systematic contribution of the ERP elicited by the cue. As a consequence, a normal averaging procedure would produce a relatively low signal/noise ratio, because the ERP elicited by the cue will add to the noise component. In addition, using a normal averaging procedure cannot estimate to what extent the cue-ERP contributed to the imperative stimulus ERP. The OPTA provides a way to estimate the contribution of linear and nonlinear components in the averaged signal that follow a gradual trend. The method is performed in the frequency domain and estimates this trend with seven polynomials, describing the ERP in a mean, and a linear up to seventh order trend to explain the variance that is included in the signal. Only those components are included that exceed a prespecified significance level $(\alpha<0.10$ (Woestenburg et al., 1983)). One result of this procedure is that the contribution of noise in the new average will be reduced to a larger extent. It will be possible to estimate both the ERP elicited by the cue and the ERP elicited by the target. The trend to be estimated and removed with OPTA is caused by sorting of the averages of the different conditions per subject, assuming that the ERP to the cue does not depend on SOA.

Per subject, three different OPTAs were employed on data for both eccentricities. In the first analysis, the signal was described with a mean factor and the linear component up to seventh order polynomial. As a consequence the new average consists of both the linear and nonlinear contribution of the cue, and the mean contribution of the target. In the second analysis, the mean contribution of the target was set to zero, which provides an estimate of the ERP elicited by the cue only. In the third analysis, we estimated only the mean component, which produces the average of the ERP elicited by the array.

Next, statistical analyses were performed over all subjects by dividing the ERPs elicited by the array into subsequent time slices (Woestenburg et al., 1992). A sample period of $20 \mathrm{~ms}$ was used for a window extending $0-800 \mathrm{~ms}$ after the onset of the imperative stimulus. Analyses were performed on lateralization, and effects of eccentricity on contralateral and ipsilateral electrodes. 


\section{Results}

In this section we will first show the contribution from the cue to the ERP elicited by the arrays, and the results of an additional checking procedure for vertical and horizontal eye movements. Secondly, we will present the results for the behavioral measures, and the results for a positive ERP component peaking at $150 \mathrm{~ms}$ (P150), and a negative ERP component peaking at $230 \mathrm{~ms}$ (N230). Significant effects are indicated with asterisks, as follows: ${ }^{*} P<0.05,{ }^{* *} P<0.01$, and ${ }^{* * *} P<0.005$.

\subsection{Contribution from the cue to the ERP elicited by the arrays}

An example of an ERP containing both the contribution of the cue and the array is presented for the occipitotemporal electrodes in the upper part of Fig. 2 for a short (i.e. left) and a long SOA (i.e. right). The middle part of Fig. 2 shows the separate contribution of the cue, and the lower part shows the residual ERP after removal of the contribution of the cue to the ERP evoked by the array.

\subsection{Checking for eye movements}

Trials containing relatively large horizontal and vertical eye movements that occurred within a window from $0-400 \mathrm{~ms}$ after presentation of the array, were excluded from analysis $(1.8 \%)$. However, it may be possible that small eye movements occurred within this interval. Therefore, an average per subject per eccentricity was computed for both HEOG and VEOG (Fig. 4). A time slice analysis, with a sample size of $20 \mathrm{~ms}$, was performed on HEOG up to $800 \mathrm{~ms}$ after the onset of the imperative stimulus. We observed horizontal eye movements in the conditions with targets presented at $3.1^{\circ}, 540 \mathrm{~ms}$ after the onset of the array, $F(1,7)=7.0^{*}$. In the conditions with targets presented at $0.7^{\circ}$ we observed horizontal eye movements $540 \mathrm{~ms}$ after the onset of the array, $F(1,7)=6.6^{*}$. Thus, it may be concluded that no significant contribution of eye movements was observed within $0-400 \mathrm{~ms}$ after the onset of the target.

\subsection{Behavioral measures}

Mean reaction times and proportion errors for both eccentricities are presented in Fig. 3. Univariate analyses were performed to estimate the effect of SOA and eccentricity. Reactions were faster to targets presented at $0.7^{\circ}$ as compared to $3.1^{\circ}$, $F(1,7)=38.6^{* * *}$. Reactions also showed a linear decrease, $F(1,7)=113.2^{* * *}$, and a quadratic increase, $F(1,7)=12.1^{* *}$, when SOA increases. More errors were made with targets at $3.1^{\circ}$ as compared to $0.7^{\circ}, t(7)=3.1^{* * *}$.

\subsection{The $P 150$}

Fig. 4 shows a positive ERP component on parietal, temporal, occipitotemporal, and occipital sites peaking at $150 \mathrm{~ms}$ after the onset of the array, which we called 


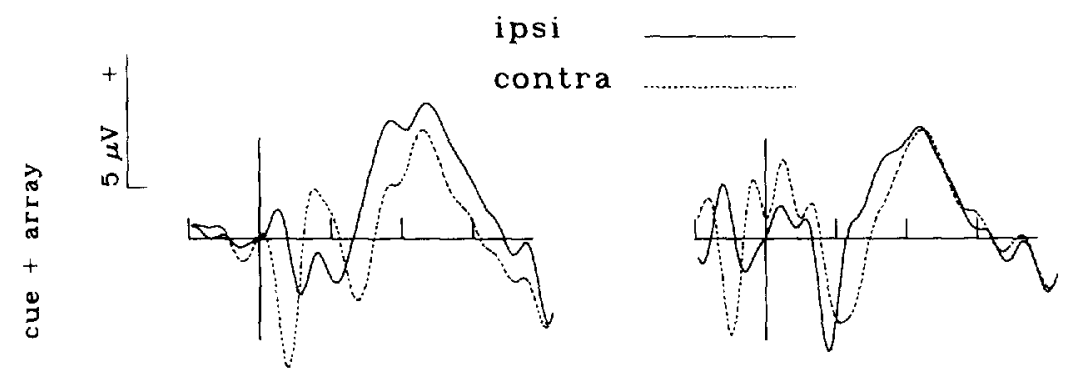

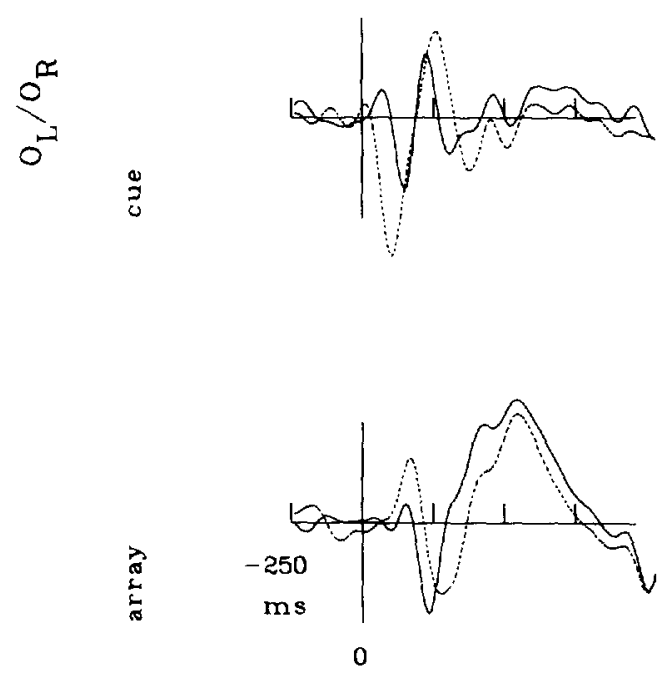

SOA $100 \mathrm{~ms}$
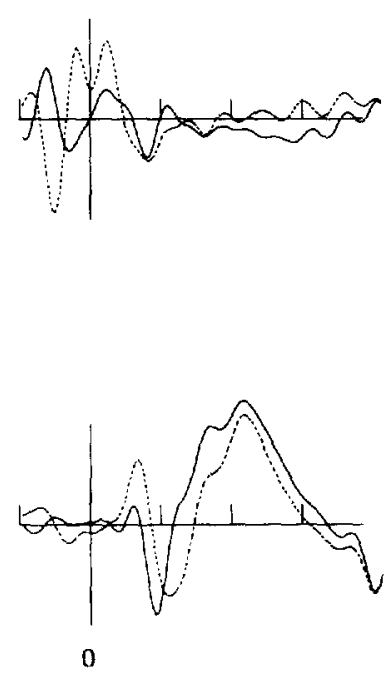

SOA $300 \mathrm{~ms}$

Fig. 2. Contralateral and ipsilateral ERPs on the occipitotemporal electrodes for targets presented at $3.1^{\circ}$ with an SOA of, respectively, 100 (left panel) and $300 \mathrm{~ms}$ (right panel). The upper panel shows the mean contribution of the array plus the linear and nonlinear contribution of the cue, the middle panel shows the linear and nonlinear contribution of the cue, and the lower panel shows the estimated mean elicited by the array corrected for the contribution of the cue.

the P150. The P150 was tested for lateralization and an effect of eccentricity. A time slice analysis (slices of $20 \mathrm{~ms}$ ) was performed for the ipsilateral and contralateral site of the occipital (O1 and O2), occipitotemporal (OL and OR), temporal (T5 and T6), parietal (P3 and P4), central (C3 and C4), and frontal (F3 and F4) electrodes. The main results are presented in Table 1.

A significant effect of eccentricity was found for the ipsilateral site on: (a) the occipitotemporal electrodes, $F(1,7)=16.3^{* *}, 1.4 \mu \mathrm{V}$; (b) the temporal electrodes, 
$F(1,7)=25.5^{* * *}, 1.1 \mu \mathrm{V}$; and (c) the parietal electrodes, $F(1,7)=16.6^{* * *}, 0.9 \mu \mathrm{V}$. The P150 was relatively large with targets presented at $0.7^{\circ}$, and relatively small with targets presented at $3.1^{\circ}$. No effects were found for the contralateral electrodes.

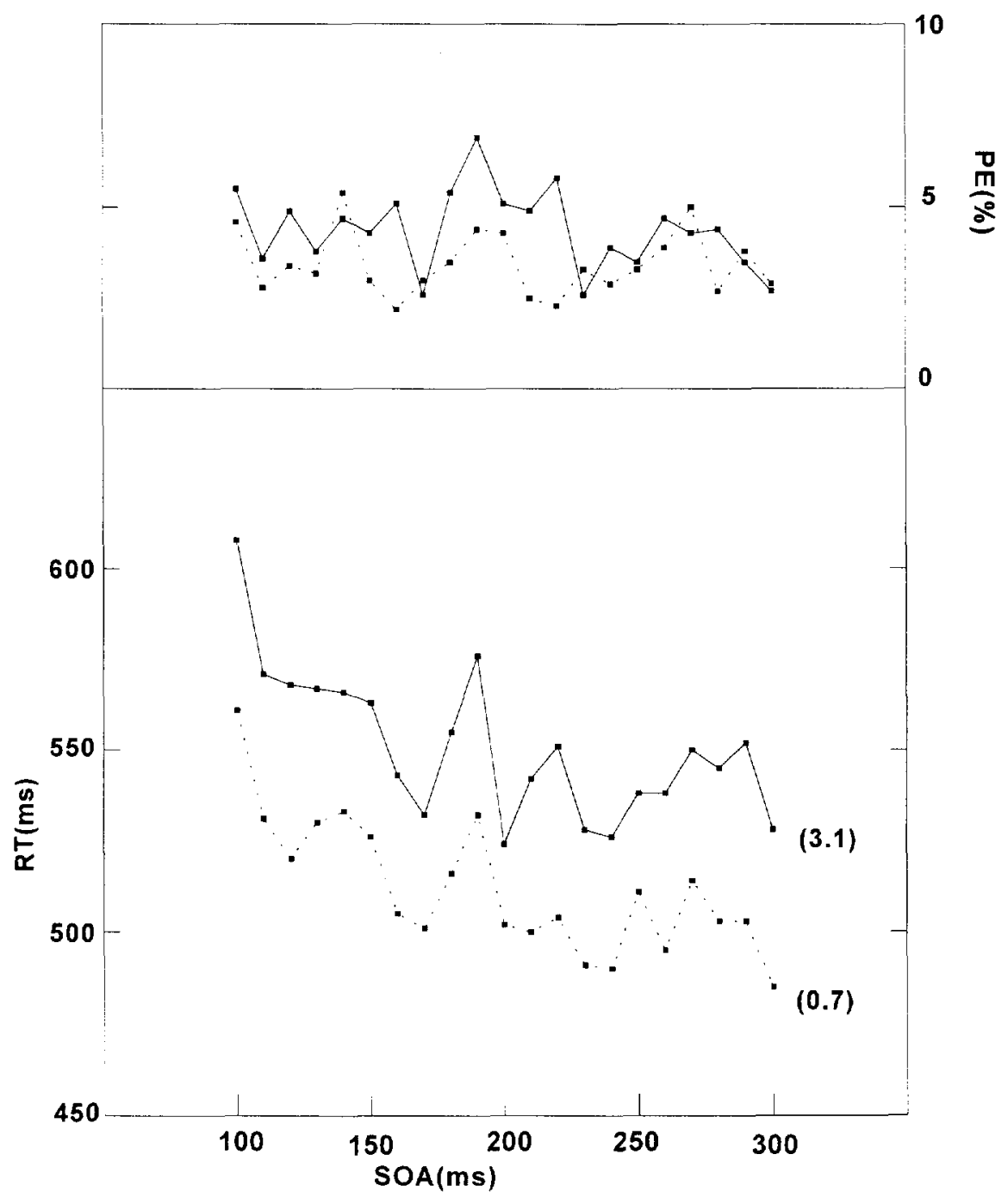

Fig. 3. Mean reaction times (RT, the lower panel) and proportion errors (PE, the upper panel) for targets presented at $3.1^{\circ}$ and $0.7^{\circ}$ from a fixation point, with a cue-target interval (SOA) that varied between 100 and $300 \mathrm{~ms}$. 


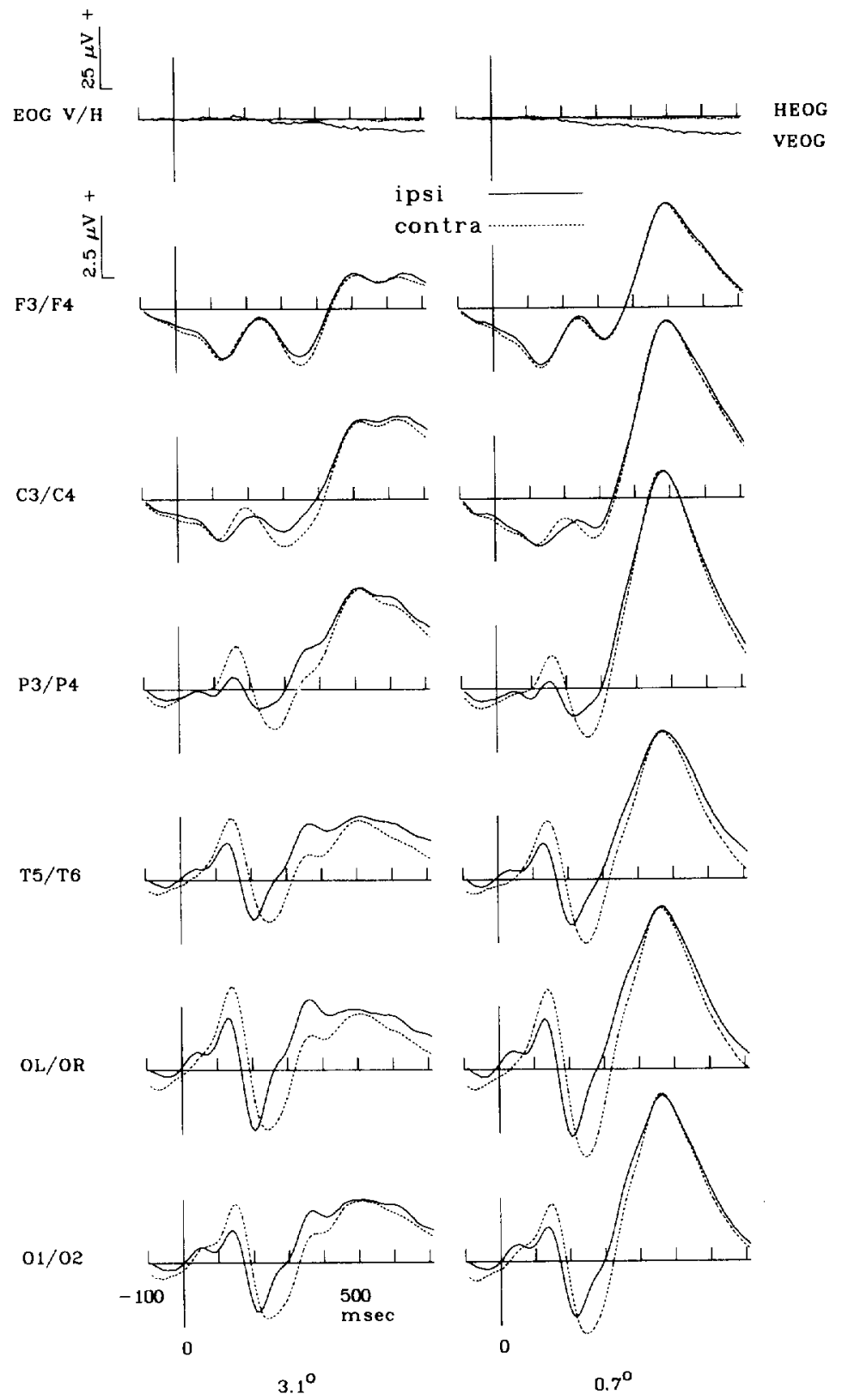

Fig. 4. VEOG and HEOG for trials with targets presented at $3.1^{\circ}$ and $0.7^{\circ}$, and ERPs elicited by the array corrected for linear and nonlinear contribution from the cue at posterior and anterior sites. For the ERPs, a comparison is made between activity at the ipsilateral (ipsi) and the contralateral (contra) hemisphere for targets presented at $3.1^{\circ}$ (left panel) and $0.7^{\circ}$ (right panel). 
Table 1

A comparison between contralateral and ipsilateral activity for the positive component peaking at approximately $150 \mathrm{msec}$, when targets were presented at $3.1^{\circ}$ and $0.7^{\circ}, F(1,7)$. A positive difference $(\Delta \mu \mathrm{V})$ indicates that contralateral activity was higher than ipsilateral activity

\begin{tabular}{|c|c|c|c|c|c|c|}
\hline \multirow[t]{2}{*}{ Electrodes } & \multicolumn{3}{|l|}{$3.1^{\circ}$} & \multicolumn{3}{|l|}{$0.7^{\circ}$} \\
\hline & Component & $F$-value & $\Delta \mu \mathrm{V}$ & Component & $F$-value & $\Delta \mu \mathrm{V}$ \\
\hline $\mathrm{O} 1 / \mathrm{O} 2$ & P150 & $56.4^{* * *}$ & 1.5 & P150 & $26.2^{* * *}$ & 0.8 \\
\hline $\mathrm{OL} / \mathrm{OR}$ & P150 & $37.2 * * *$ & 2.6 & P150 & $75.7 * * *$ & 1.5 \\
\hline T5 & P150 & $46.6^{* * *}$ & 2.2 & P150 & $57.1^{* * *}$ & 1.3 \\
\hline P3/P4 & $\mathrm{P} 150$ & $52.3^{* * *}$ & 1.5 & $\mathrm{P} 150$ & $57.1 * * *$ & 0.7 \\
\hline $\mathrm{C} 3 / \mathrm{C} 4$ & $\mathrm{P} 200$ & $16.4^{* * *}$ & 0.7 & P180 & $18.3^{* * *}$ & 0.4 \\
\hline $\mathrm{F} 3 / \mathrm{F} 4$ & P240 & n.s. & 0.0 & $\mathrm{P} 240$ & n.s. & 0.0 \\
\hline
\end{tabular}

\subsection{The $N 230$}

Fig. 4 shows a negative component at parietal, temporal, occipitotemporal, and occipital electrodes peaking at about $230 \mathrm{~ms}$, which we called the $\mathrm{N} 230$. Again, a time slice analysis was used. However, we decided to report the time interval of significant differences (e.g. $240-300 \mathrm{~ms}$ ) because maximal differences were not observed at peak amplitudes. We will report the minimal and maximal $F$-value within this interval (e.g. $9 \leq \mathrm{F}(1,7) \leq 12$ ), and the minimal and maximal amplitude differences (e.g. $0.5-2.1 \mu \mathrm{V}$ ). The main results are presented in Table 2

No differences in the effect of eccentricity were observed for the occipital, occipitotemporal, temporal, and parietal electrodes. However, for the central electrodes a greater negativity was found with targets on $3.1^{\circ}$ as compared to $0.7^{\circ}$

Table 2

A comparison between contralateral and ipsilateral activity for the negative component peaking at approximately $230 \mathrm{msec}$, when targets were presented at $3.1^{\circ}$ and $0.7^{\circ}, F(1,7)$. Intervals are reported in which significant effects were observed. We reported the minimal and maximal $F$-values. In addition, the minimal and maximal differences in $\mu \mathrm{V}$ are reported. A positive difference $(\Delta \mu \mathrm{V})$ indicates that contralateral activity was higher than ipsilateral activity

\begin{tabular}{|c|c|c|c|c|c|c|c|c|}
\hline \multirow[t]{3}{*}{ Electrodes } & \multicolumn{4}{|l|}{$3.1^{\circ}$} & \multicolumn{4}{|l|}{$0.7^{\circ}$} \\
\hline & \multicolumn{3}{|l|}{$F$-value } & \multirow[t]{2}{*}{$\Delta \mu \mathrm{V}$} & \multicolumn{3}{|l|}{$F$-value } & \multirow[t]{2}{*}{$\Delta \mu \mathrm{V}$} \\
\hline & Interval & $\min$ & $\max$ & & Interval & $\min$ & $\max$ & \\
\hline $\mathrm{O} 1 / \mathrm{O}_{2}$ & $240-300$ & $8.8^{*}$ & $20.2^{* * *}$ & $1.3-1.9$ & $240-300$ & $23.8^{*}$ & $57.9^{* * *}$ & $0.7-1.8$ \\
\hline $\mathrm{OL} / \mathrm{OR}$ & $260-300$ & $11.8^{* *}$ & $18.0^{* * *}$ & $2.2-2.4$ & $240-300$ & $9.4^{*}$ & $34.1 * * *$ & $0.9-2.4$ \\
\hline T5/T6 & $260 \cdots 300$ & $7.4^{*}$ & $11.5^{* *}$ & $1.3-1.5$ & $260 \ldots 300$ & $14.1^{* *}$ & $31.0^{* * *}$ & $0.6-1.5$ \\
\hline $\mathrm{P} 3 / \mathrm{P} 4$ & $260-300$ & $12.1^{* *}$ & $33.8^{* * *}$ & $1.2-1.4$ & $260-280$ & $19.7^{* * *}$ & $53.4^{* * *}$ & $1.0-1.1$ \\
\hline $\mathrm{C} 3 / \mathrm{C} 4$ & $280-340$ & $6.0^{*}$ & $7.8^{*}$ & $0.4-0.6$ & $260-300$ & $6.1^{*}$ & $20.9 * * *$ & $0.2-0.4$ \\
\hline $\mathrm{F} 3 / \mathrm{F} 4$ & - & - & - & - & $\cdot-$ & - & - & - \\
\hline
\end{tabular}


on both the ipsilateral $\left(280-320 \mathrm{~ms}, 15.5^{* *} \leq F(1,7) \leq 128.8^{* * *}\right)$, and the contralateral electrodes $\left(260-300 \mathrm{~ms}, 7.2^{*} \leq F(1,7) \leq 68.3^{* * *}\right)$. Similar results were found on the frontal electrodes; ipsilateral $\left(280-340 \mathrm{~ms}, 13.9^{* *} \leq F(1,7) \leq\right.$ $\left.49.7^{* * *}\right)$, and contralateral $\left(280-360 \mathrm{~ms}, 16.6^{* *} \leq F(1,7) \leq 60.8^{* * *}\right)$.

\section{Discussion}

Several authors have suggested that attentional resources can be allocated at an early sensory processing level. Therefore, the question was raised whether or not voluntary and involuntary allocation of attentional resources as induced by respectively symbolic and peripheral cues, invoke the same level of sensory processing.

Hillyard et al. (1994) performed an ERP study in which they observed a P1 enhancement for symbolicly cued targets but not for peripherally cued targets. They suggested that peripheral and symbolic cues differ in their capacity to invoke the earliest form of attentional selection. However, several studies showed that the optimal effect of peripheral cues is observed with much shorter SOAs than the SOAs used by Hillyard et al. We performed an experiment with $100 \%$ valid peripheral cues that preceded a multi-element array with laterally presented targets with an SOA between 100 and $300 \mathrm{~ms}$. Target identity determined the appropriate response. ERPs elicited by the array were corrected for contribution from the cue by using the OPTA. In addition, trials containing detectable eye movements were removed. Analysis of HEOG of the accepted trials showed no significant horizontal eye movements until $540 \mathrm{~ms}$ after onset of the array.

Behavioral measures showed an improvement in performance when SOA increased from 100 to $300 \mathrm{~ms}$. These results suggest that IOR (inhibition of return), as reported by Posner \& Cohen (1984), is not involved within this relatively short interval. In addition, performance improved for targets presented at $0.7^{\circ}$ as compared to targets presented at $3.1^{\circ}$, which can be ascribed to an increase in visibility.

An estimation of the ERP elicited by the array, corrected for linear and nonlinear contribution of the cue, showed an enhancement of a P150 component on the contralateral as compared to the ipsilateral hemisphere on posterior (i.e., occipital, occipitotemporal, temporal and parietal) sites. This lateralization was apparent when targets were presented on both $3.1^{\circ}$ and $0.7^{\circ}$. For central electrodes a delay was observed as compared to the posterior sites. When targets were presented at $3.1^{\circ}$ and $0.7^{\circ}$ the delay was 50 and $30 \mathrm{~ms}$ respectively. For central electrodes, the delayed component was lateralized for both eccentricities. For frontal electrodes a further nonlateralized delay was observed, resulting in a peak at about $240 \mathrm{~ms}$. The amplitude of the P150 seems to be sensitive to spatial information provided by the cue. This suggests that peripheral cues influence a very early stage in visual information processing. If we assume that occipital electrodes mainly pick up activity from prestriate areas, then it may be suggested that peripheral cues modulate processing at the level of the prestriate cortex. Another finding was an effect of eccentricity that was maximal at the temporal electrodes. A main differ- 
ence appeared on the ipsilateral hemisphere; an amplitude reduction was manifest with targets presented on $3.1^{\circ}$ as compared to $0.7^{\circ}$. This suggests that activation of the irrelevant side diminishes when attention is focused at $3.1^{\circ}$ than to $0.7^{\circ}$. This may be attributed to the joint involvement of both hemispheres in processing of a more centrally presented target. Thus, in contrast to the experiments performed by Hillyard et al. (1994), we observed an effect on P1 amplitude with peripheral cues. In our study, the Pl component appeared somewhat later, which may be attributed to the use of different stimuli.

The use of long SOAs in the peripheral cue condition of Hillyard et al. can lead to inhibition of return (IOR). This could be the reason why they did not observe an attention effect on the P1 component. However, their behavioral measures showed a benefit for attended as compared to unattended locations, which suggests that IOR was not present in their task. Müller \& Rabbitt (1989) argued that IOR may be suppressed by voluntary orienting. This suppression might have an effect on the level at which attentional resources are allocated. Thus, on the basis of modulation of the P1 component in our experiment, it may be suggested that both voluntary and involuntary allocation of resources invoke about the same level of sensory processing.

We observed larger negativity at the contralateral as compared to the ipsilateral site for the $\mathrm{N} 230$, after the posterior contralateral $\mathrm{N} 230$ reached its maximal amplitude. The difference began at about $240 \mathrm{~ms}$ and lasted until the offset of the N230. It must be noted that this difference can be interpreted as both a latency effect and an amplitude effect. The difference may be interpreted as the prolongation of an elaboration process that acts upon the selected element. Furthermore, the peak was delayed for about $80 \mathrm{~ms}$ on the anterior site as compared to the posterior site. A contralateral enhancement was found at the central electrodes, which started at $280 \mathrm{~ms}$. A nonlateralized enhancement for the N230 appeared on the anterior site when targets were presented at $3.1^{\circ}$ as compared to $0.7^{\circ}$. This enhancement was present from about 300 to $380 \mathrm{~ms}$, and may be interpreted as the insertion of a specific process when targets are presented at $3.1^{\circ}$.

Mangun \& Hillyard (1991) argued that an enhancement of the N1 component might reflect reorienting, or reallocation of attentional resources. However, they observed the N1 component between 150 and $200 \mathrm{~ms}$, whereas in our study it was observed much later. To avoid complicating interpretations we assume that a specific process is prolonged at the contralateral posterior site. In addition, the nonlateralized anterior enlargement for targets presented at $3.1^{\circ}$ as compared to targets presented at $0.7^{\circ}$ might reflect a process that is more active if a deviant array-element is presented more eccentricly.

\section{Conclusions}

We suspected that the reason for not previously finding an early positive enlargement with peripheral cues might be the use of too long SOAs. Therefore, an experiment was performed with short SOAs. The OPTA was performed to remove 
the ERP elicited by the cue from the ERP elicited by the array. The adjusted ERP for the array clearly showed a contralateral enhancement of the P1 component at posterior electrodes, which confirms the idea that the null findings of Mangun and Hillyard may be attributed to the use of long SOAs. Hence, the P1 enhancement at contralateral electrodes might reflect both voluntary and involuntary allocation of resources to a specific location.

\section{Acknowledgements}

This research was supported by a grant from the Dutch Organization for Fundamental Research (NWO) (No. 560-265-056). We would like to thank Annekee Bos for collecting a major part of the data, and we are grateful for comments on an earlier draft of this manuscript made by Art Kramer and an anonymous reviewer.

\section{References}

Campbell, \& Bell (1992). InstEP systems. InstEP corp. Ottawa, Canada.

Duncan, J. (1980). The locus of interference in the perception of simultaneous stimuli. Psychological Review, 87, 272-300.

Eimer, M. (1994). An ERP study on visual spatial priming with position focused onsets. Psychophysio/ogy, 31, 154-163.

Hawkins, H.L., Hillyard, S.A., Luck, S.J., Mouloua, M., Downing, C.J., \& Woodward, D.P. (1990). Visual attention modulates signal detectability. Journal of Experimental Psychology: Human Perception and Performance, 16, 802-811.

Heinze, H.J., Luck, S.J., Mangun, G.R., \& Hillyard, S.A. (1990). Visual event-related potentials index focused attention within bilateral stimulus arrays. I. Evidence for early selection. Electroencephalography and Clinical Neurophysiology, 75, 511-527.

Hillyard, S.A., Luck, S.J., \& Mangun, G.R. (1994). The cuing of attention to visual field locations: analysis with ERP recordings. In H.J. Heinze, T.F. Münte, G.R. Mangun (Eds.), Cognitive Electrophysiology, (pp. 1-25). Boston, MA: Birkhäuser.

Jonides, J. (1981). Voluntary versus automatic control over the mind's eye movement. In J.B. Long and A.D. Baddely (Eds.), Attention and Performance, Vol. IX, (pp. 187-203). Hillsdale, NJ: Lawrence Erlbaum.

Jonides, J. (1983). Further toward a model of the mind's eye's movement. Bulletin of the Psychonomic Society, 21, 247-250.

Mangun, G.R., \& Hillyard, S.A. (1990). Allocation of visual attention to spatial locations: Tradeoff functions for event-related brain potentials and detection performance. Perception \& Psychophysics, $47,532-550$.

Mangun, G.R., \& Hillyard, S.A. (1991). Modulations of sensory-evoked brain potentials indicate changes in perceptual processing during visual-spatial priming. Journal of Experimental Psychology: Human Perception and Performance, 17, 1057-1074.

Müller, H.J., \& Rabbitt, P.M.A. (1989). Reflexive and voluntary orienting of visual attention: time course of activation and resistance to interruption. Journal of Experimental Psychology: Human Perception and Performance, 15, 315-330.

Posner, M.I. (1980). Orienting of attention: The VIIth Sir Frederic Bartlett Lecture. Quarterly Journal of Experimental Psychology, 32, 3-25. 
Posner, M.I., Snyder, C.R.R., \& Davidson, B.J. (1980). Attention and the Detection of Signals. Journal of Experimental Psychology: General, I09, 160-174.

Posner, M.I., \& Cohen, Y. (1984). Components of visual orienting. In H. Bouma and D.G. Bouwhuis (Eds.), Attention and Performance, Vol. X. (pp. 531-556). Hillsdale, NJ: Lawrence Erlbaum.

Shaw, M.L. (1984). Division of attention among spatial locations: a fundamental difference between detection of letters and detection of luminance increments. In H. Bouma \& D.G. Bouwhuis (Eds.), Attention and Performance, Vol. X, (pp. 109-121) Hillsdale, NJ: Lawrence Erlbaum.

Van der Heijden, A.H.C. (1992). Selective Attention in Vision. London: Routledge.

Woestenburg, J.C., Verbaten, M.N., Hees, van H.H., \& Slangen, J.L. (1983). Single trial ERP estimation in the frequency domain using othogonal polynomial trend analysis (OPTA): estimation of individual habituation. Biological Psychology, 17, 173-191.

Woestenburg, J.C., Das-Smaal, E.A., Brand, E., \& Kramer, S. (1992). Learning during visual search in children with attentional and learning problems: a trial-to-trial evaluation of RT and ERP measures. Journal of Psychophysiology, 6, 204-224.

Woestenburg, J.C. (1994). Statistical Time Series Package for Biological Signals (STPBS). Bussum, The Netherlands: *Talo b.v.

Woldorff, M.G. (1993). Distortion of ERP averages due to overlap from temporally adjacent ERPs: analysis and correction. Psychophysiology, 30, 98-119. 